\title{
Sampling for IPM Decision Making: Where Should We Invest Time and Resources?
}

\author{
Jan P. Nyrop, Michael R. Binns, and Wopke van der Werf
}

First author: Department of Entomology, Cornell University, NYSAES, Geneva, NY 14456; second author: 1 Findlay Avenue, Ottawa, Ontario K1S 2T8 Canada; and third author: Department of Theoretical Production Ecology, Wageningen Agricultural University, Bornsesteeg 47, Wageningen, 6708PD, the Netherlands.

Accepted for publication 13 July 1999.

ABSTRACT

\begin{abstract}
Nyrop, J. P., Binns, M. R., and van der Werf, W. 1999. Sampling for IPM decision making: Where should we invest time and resources? Phytopathology 89:1104-1111.

Guides for making crop protection decisions based on assessments of pest abundance or incidence are cornerstones of many integrated pest management systems. Much research has been devoted to developing sample plans for use in these guides. The development of sampling plans has usually focused on collecting information on the sampling distribution of the pest, describing this sampling distribution with a mathematical model, formulating a sample plan, and sometimes, but not always, evaluating the performance of the proposed sample plan. For crop protection decision making, classification of density or incidence is usually more
\end{abstract}

appropriate than estimation. When classification is done, the average outcome of classification (the operating characteristic) is frequently robust to large changes in the sampling distribution, including estimates of the variance of pest counts, and to sample size. In contrast, the critical density, or critical incidence, about which classifications are made, has a large influence on the operating characteristic. We suggest that rather than investing resources in elaborate descriptions of sampling distributions, or in fine-tuning sample size to achieve desired levels of precision, greater emphasis should be placed on characterizing pest densities that signal the need for management action and on designing decision guides that will be adopted by practitioners.

Additional keyword: probability.
There can be little doubt that basing pest management decisions on the current state of a pest population is, in many cases, a sound strategy. There are two reasons for making this assertion. First, such a strategy can and has been used to reduce pesticide use, which, in turn, can reduce pest control costs, can be an important first step toward biological control, and may be a component of a resistance management strategy. Secondly, if pest management tactics such as biological control or cultural practices fail, pest control can still be accomplished through remedial actions, and decision guides should be available to determine when such actions are needed.

A key component of integrated pest management (IPM) decision guides are sampling plans that prescribe how and when samples should be taken to assess the pest population. During the past 30 years, scores of sample plans have been devised and described in the literature (references in literature citation 14). Developing these sample plans has usually entailed collecting information on the distribution of sample observations, describing this sampling distribution with a mathematical model, formulating a sample plan, and sometimes, but not always, evaluating the performance of the proposed sample plan. An important question is whether developing these sampling plans using this scenario is a wise investment of time and resources.

In this paper, we will argue that precise descriptions of sampling distributions are often unnecessary and that generic descriptions will likely suffice. This is not a novel insight. Green (6) and Jones (9) came to similar conclusions, though from the perspective of

Corresponding author: J. P. Nyrop; E-mail address: jpn2@ cornell.edu

Publication no. P-1999-0930-010

(c) 1999 The American Phytopathological Society estimating abundance rather than from the view of sampling for decision making. Elliot et al. (5) also suggested that generic models of sample distributions might suffice for developing sampling plans for pests of wheat and rice. From the perspective of sampling for decision making, we think an even stronger case can be made for the use of generic models of sample distributions. We will also suggest that specification of sample size so that some specified precision in the sample information is obtained is usually unnecessary. Rather, minimal sample sizes should be established so that the risks of incorrect decisions are acceptable and sample plans designed so that the sample information is representative of the pest population. Rather than investing resources in detailed descriptions of sampling distributions or in fine-tuning sample plans to achieve desired levels of precision, greater emphasis should be placed on characterizing pest densities that signal the need for management intervention and on designing decision guides that will be adopted by practitioners.

We develop our thesis in three stages. In the first stage, sampling for decision making is defined and the crucial roles of operating characteristic (OC) and average sample number (ASN) functions in designing sampling plans are described. In the second stage, the influences of sample plan parameters on OC and ASN functions are elucidated and conclusions about the importance of correctly describing these parameters are drawn. Finally, we suggest how resources should be allocated when developing sampling plans for IPM decision making.

\section{SAMPLING FOR DECISION MAKING}

Initially, one might think a really precise and accurate estimate of pest density would be useful for making a decision on the need 
for some pest management action. With an estimate of pest abundance, a grower could weigh all relevant information and come to a decision on the need for control. However, it is rare that methods exist for combining all relevant pieces of information pertaining to a pest's impact on a crop for the purpose of deciding on the need for corrective action. Usually, all that can be made use of, and hence is required about pest abundance, is knowledge of whether pest density or incidence falls into one of two or perhaps more categories. This is because the understanding and models that might make use of all relevant information to arrive at a crop protection decision, for the most part, do not exist. Thus, it usually suffices to know whether pest abundance is less than some critical level and control is not needed or if density is greater than the critical level and control is warranted. Because the information contained in a precise estimate of density can usually not be fully used in pest management decision making, density estimation is often not needed or appropriate in this context.

But is it mere rhetoric to suggest that estimation and classification are different? Operationally, estimation and classification are rather similar. In both cases, samples must be collected, pests organisms must be counted, and statistics must be calculated. The distinction between estimation and classification is manifest in the purpose for which the sample data are collected, and the difference in purpose justifies and, in some cases even directs, differences in the design of sampling protocols. Estimation procedures are fuelled by the questions: how many samples are required so that an estimate is within some range of a true value, or how many samples are required so that an estimate has a specified level of precision? In pest management, information on pest abundance is most often used to direct one of two or more pest management actions. Therefore, for pest management purposes, it is usually most informative to ask how many samples are required so that the likelihoods of incorrect management decisions are acceptable?

When there are only two possible decisions to choose from, densities below some critical density, $c d$, dictate one decision, and densities greater than $c d$ dictate the other. In such cases, the likelihood of making either decision based on sample data is obtained from the probability of classifying pest density as less than or equal to $c d$ given the true density. These likelihoods comprise the OC function for a sample plan. If pest incidence is the quantity measured, critical proportion, or $c p$, is used in lieu of $c d$. We use the terms $c d$ or $c p$ rather than economic injury level or action threshold because, while $c d$ or $c p$ may be based on these quantities, they may reflect other criteria as well.

Because sample information contains uncertainty, sample data can lead to an incorrect decision. However, as long as the sample data are not biased, as sample size increases, the likelihood of an incorrect decision decreases. The objective when designing sampling protocols for use in pest management is to balance the cost of data collection with the likelihood of making an incorrect decision. This trade-off cannot be studied when the cost of data collection is keyed to the precision of an estimate. Therefore, OC and ASN functions should usually be used to judge the performance of sample plans used in pest management decision making. An example will illustrate this.

Suppose a decision should be made to treat a pest population if the density exceeds 5 , so $c d=5$. Suppose also that the variance of sample counts of the pest population can be modelled as a function of the mean using Taylor's power law (TPL) (16) as

$$
\sigma^{2}=a \mu^{b}
$$

with $a=1.5$ and $b=2$. The precision of an estimated mean can be expressed as the coefficient of variation $(C V)$, which is the ratio of the standard deviation of the mean to the mean.

$$
\begin{aligned}
C V & =\frac{\sqrt{\frac{\sigma^{2}}{n}}}{\mu} \\
& =\frac{\sigma_{\mu}}{\mu}
\end{aligned}
$$

By specifying $C V$ and by replacing $\sigma^{2}$ with equation 1 , a required sample size can be determined for estimating a mean for a population with any assumed true mean.

Now suppose we will sample a pest population so that a precision of $C V=0.2$ is obtained. This tells us nothing of the likelihood that a decision to treat or not treat will be made given any true population mean. This likelihood can, however, be easily determined. Assuming that the central limit theorem applies so that sample means are normally distributed, the $\mathrm{OC}$ function can be calculated as

$$
\begin{aligned}
\text { OC } & =P\left(\hat{\mu} \leq c d \mid \mu, \sigma_{\mu}\right) \\
& =P\left(\frac{\hat{\mu}-\mu}{\sigma_{\mu}} \leq \frac{c d-\mu}{\sigma_{\mu}}\right) \\
& =P\left(z \leq \frac{c d-\mu}{\sigma_{\mu}}\right)
\end{aligned}
$$

in which $P(\bullet)$ denotes the probability of the event within the parentheses and $z$ is a standard normal deviate. By specifying $C V$, required sample sizes can be determined for each true mean and the corresponding OC calculated.

Should a $C V$ of 0.2 or 0.15 be used for making pest management decisions for this hypothetical pest? There is no way to answer this question with the information at hand, despite the fact that $C V \mathrm{~s}$ appropriate for decision making have been endorsed (15). It is more informative to study the OC functions for prospective sampling plans and use this function in conjunction with the ASN function to decide on the acceptability of a plan. The ASN provides the average or expected number of samples required by the sample plan to classify density given the true density. When fixed sample size plans are used, the ASN is, in fact, the actual sample size. When sequential procedures are used, the ASN values are expectations and actual sample sizes may be greater or less than these values (2).

Figure 1 shows OC and ASN functions for sample plans used to classify density with respect to $c d=5$ when sample sizes are determined by requiring that a $C V$ of 0.2 or 0.15 be achieved when estimating the mean. The OC is more informative than simply knowing the precision of the sample estimate and allows sample size to be adjusted to meet desired levels of risk. For true densities less than 5, the OC provides the probability of correctly deciding to do nothing. At densities greater than 5 , the OC provides the probability of incorrectly deciding to do nothing. In this case, little
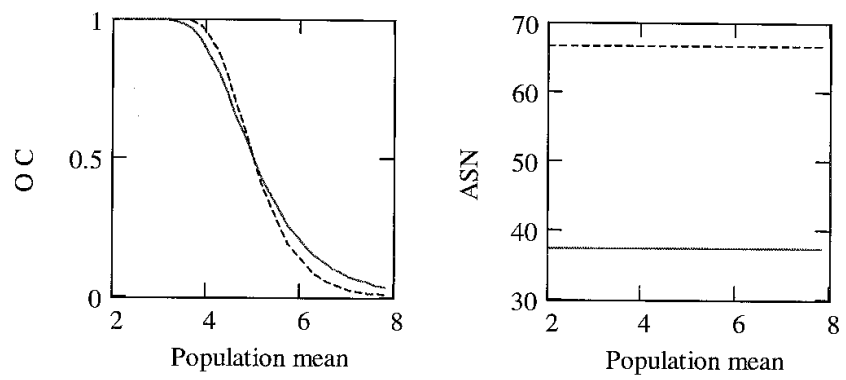

Fig. 1. Operating characteristic (OC) and average sample number (ASN) functions for a sample plan designed to achieve a coefficient of variation $(\mathrm{CV})$ of 0.2 (solid line) or 0.15 (dashed line) and used to classify density with respect to critical density $(c d)=5$. The variance was specified as $\sigma^{2}=1.5 \mu^{2}$. 
might be gained by using $C V=0.15$, because the reduction in risk of an incorrect decision is rather slight, but sample sizes are nearly doubled. Whether or not the reduction in risk associated with $C V=$ 0.15 is worthwhile depends on the costs of making an incorrect decision and the costs of collecting and processing samples. In some cases, even though sample sizes are nearly doubled, this additional investment might be insignificant if the costs of incorrect decisions are high or if samples can be collected and processed quickly. The most important point here is that these assessments cannot be made when sampling plans used for decision making purposes are based solely on the precision of the estimated mean, but depend on the $\mathrm{OC}$ and $\mathrm{ASN}$ functions.

One might argue that in more complicated situations in which sample information is used to decide among more than two management options, the OC function and classification are not appropriate and, hence, estimation must be used. This is incorrect. It is possible to generate an $\mathrm{OC}$ function for all possible management options; although, to keep the terminology clear, OC functions are renamed probability of decision (PDEC) functions. Even if density estimates are used in a model to forecast pest populations and management decisions are based on these forecasts, it is still possible to generate PDEC functions. Generation of these PDEC functions is illustrated below, in which a simple exponential growth function is used to forecast future pest density. Simulation must now be used to estimate the PDEC functions (4).

Assume that we are now interested in deciding among three management actions. If the forecasted pest density is less than or equal to $c d_{1}$, action 1 is taken; if the forecasted pest density is greater than $c d_{1}$ and less than or equal to $c d_{2}$, action 2 is taken; and if the predicted pest density exceeds $c d_{2}$, action 3 is taken. We assume that uncertainty about the forecasted density arises solely from the estimate from which a forecast is made. This assumption is unrealistic, but does not affect the point we wish to make. Density is forecasted 10-time steps into the future $\left(\hat{\mu}_{10}\right)$ by applying exponential growth to the estimated density $\left(\hat{\mu}_{0}\right)$

$$
\hat{\mu}_{10}=\hat{\mu}_{0} e^{0.1 \times 10}
$$

Figure 2 shows PDEC functions for two sample plans whose sample sizes yield estimated means with a $C V$ of 0.2 or 0.15 . The PDEC functions are plotted with respect to the true mean at the time of

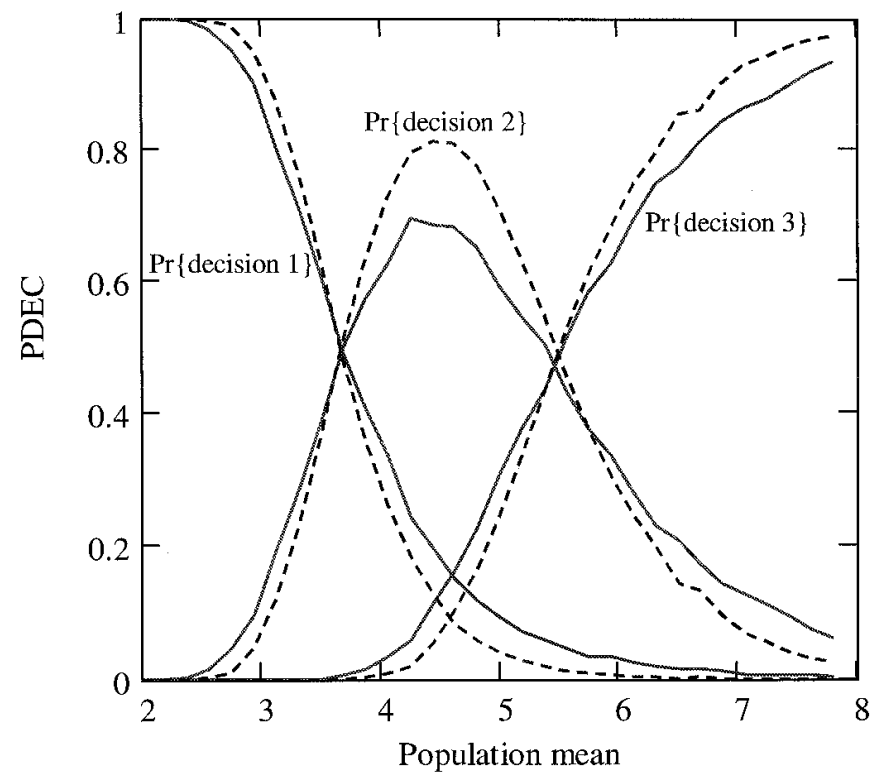

Fig. 2. Probability of decision (PDEC) functions for two sample plans used to classify forecasted density with respect to critical densities of 10 and 15 . The PDEC functions are plotted with respect to the true mean at the time of sampling. Sample sizes were selected to achieve a coefficient of variation $(\mathrm{CV})$ of 0.2 (solid line) or 0.15 (dashed line). Sample sizes are shown in Figure 1. sampling. It is evident that a small change in the coefficient of variation $(0.2$ to 0.15$)$ leads to very large changes in the required sample size (Fig. 1), which, in turn, has only a modest effect on the PDEC functions. But this would never have been discerned if a coefficient of variation had been blithely accepted.

Thus, the key distinction between estimation and classification is the purpose for which the sample information is collected. With estimation, we begin by specifying how close we wish an estimate to be to a true value. With classification, we begin by specifying how frequently we will tolerate incorrect decisions. In both cases, sample sizes are adjusted in order to achieve the specified objective. Clearly, concepts pertinent to estimation and classification are related; however, the purpose to which these concepts are applied are quite different as is the approach taken in designing sampling procedures to meet estimation or classification goals. For IPM decision making, the emphasis should be on classification. While this requires explicit use of $c d$ or $c p$ and these parameters may only be vaguely known, this is not a limitation. With estimation procedures, this knowledge is implicitly used.

\section{INFLUENCE OF SAMPLE PLAN PARAMETERS ON OC AND ASN FUNCTIONS}

Three types of parameters influence OC and ASN functions for sampling plans that classify density or proportion with respect to critical values: (i) those that describe the model for the distribution of sample observations, (ii) those that determine the shape of the stop boundary for the sample plan, and (iii) the critical density or critical proportion. Whenever a sample plan is constructed, a model is required for the sampling distribution of the sample observations. Without such models, OC and ASN functions cannot be determined and prospective sampling plans cannot be evaluated. These models may be probability distribution functions (e.g., negative binomial distribution), combinations of empirical relationships and probability distribution functions (e.g., negative binomial distribution with Taylor's power law), or collections of data from which bootstrapping (12) is done. The stop boundary for any sample plan consists of rules for determining when sampling is complete. In the simplest case, the stop boundary for a fixed sample size is but one number and sampling ceases once this many samples have been observed. With sequential sampling, the total number of organisms found in $n$ samples is compared with one or more values and sampling is either terminated or continued based on this comparison. Parameters that influence the stop boundaries alter their shape and width. The critical density or critical proportion must also be considered as a parameter of sample plans, because classification can only be done relative to some value.

The general effects of sample plan parameters on OC and ASN functions are predictable. We will use Iwao's sequential decision rule (8) to illustrate these patterns. We use Iwao's procedure because it is relatively simple to understand and has a minimal number of parameters. From the perspective of sample efficiency, Iwao's procedure is not the best, but for the purpose at hand, this is unimportant. The stop boundaries for Iwao's procedure are based on confidence intervals for an estimated mean. Assuming that the mean is equal to the critical density, $c d$ (or $c p$ ), the upper boundary is calculated as the upper confidence interval for the mean, estimated with sample size $n$, multiplied by the sample size. Multiplication by the sample size expresses the boundary in terms of a total count rather than a mean, thereby making comparisons to sample observations easier. The lower boundary is based on a lower confidence interval. The upper and lower boundaries, $U B_{n}$ and $L B_{n}$, respectively, are calculated as

$$
\begin{aligned}
& U B_{n}=n *\left(c d+z_{\alpha} * \frac{s}{\sqrt{n}}\right) \\
& L B_{n}=n *\left(c d-z_{\alpha} * \frac{s}{\sqrt{n}}\right)
\end{aligned}
$$


in which $s$ is the standard deviation of the sample counts when the true mean is equal to $c d, n$ is the sample size, and $z_{\alpha}$ is a standard normal deviate such that the probability of a larger $z=\alpha$. All parameters except $z_{\alpha}$ have readily understood interpretations; $z_{\alpha}$ is best thought of as a parameter defining how far apart the boundary lines are and should not be interpreted as an error rate or even a single point on the OC function. The standard deviation used to calculate the stop boundaries is that when the mean equals $c d$ or $c p$ and is based on the probability distribution used to describe the sample counts. As a result, Iwao stop boundaries are, in an absolute sense, predicated on a probability model for the sample counts.

The stop boundaries calculated using equation 5 are curvilinear lines that diverge more and more slowly with increasing $n$ (Fig. 3 ). If the cumulative count after $n$ samples, $S_{n}$, is greater than $U B_{n}$, sampling is stopped and density classified as greater than $c d$. Likewise, if $S_{n}$ is less than $L B_{n}$, sampling is stopped and density classified as less than or equal to $c d$. Due to practical constraints, sampling must terminate when $n$ reaches a specified maximum value, $\max n$. If $n$ reaches $\max n$, the estimate of mean density, $S_{\max n} / \max n$ is compared with $c d$. If the estimated mean is greater than $c d$, density is classified as greater than $c d$, and as less than or equal to $c d$ otherwise. Anytime $S_{n}$ is greater than $c d^{*} \max n$, the density will be classified as greater than $c d$. This results in a portion of the upper boundary being a horizontal line that ends at the point maxn, $c d^{*} \max n$ (Fig. 3). To ensure a representative sample, the stopping rule is usually not applied until a minimum number of samples units, $\min n$, is collected.

Changes in the parameters of a sample plan based on Iwao's sequential procedure and changes in the distribution of sample counts have some predictable general effects on the OC and ASN functions, but the magnitude of these effects and the interactions among parameters are not easily predicted. Increasing $z_{\alpha}$ (decreasing $\alpha$ ) increases the width of the boundary, which, in turn, increases the ASN function. The effect on the OC is to make it steeper; however, the magnitude of the change may be slight. Increasing the minimum and maximum sample sizes results in a steeper slope of the OC function and an increased ASN. Increasing $c d$ shifts the $\mathrm{OC}$ and ASN functions to the right on the mean density and sample size axes, respectively. Finally, increasing the variance of the sample observations above the variance used to construct the stop boundary flattens the OC function, but the effect on the ASN function is not easily predicted.

The patterns described above are frequently interpreted to mean that parameters for sample plans must be carefully described or determined. As a result, sampling distributions are repeatedly described in detail, even for the same pest infesting only a slightly different crop or inhabiting a different geographic region. In addition, vigilant attention is often paid to ensuring that realized OC and ASN values do not deviate from those specified during sample plan design. Finally, debates are waged over the best and most accurate way of describing sample observations for the purpose of designing and evaluating sample plans (e.g., bootstrapping versus simulation).

Each of these undertakings entails an investment of resources and time. Are these the most profitable pursuits when the goal is to develop sample plans for use in IPM decision making? We think not and instead propose that generic sample distribution models be used to initiate sample plan design, that sample size determination be based more on achieving a representative sample rather than on a somewhat arbitrary specification of classification precision, that far greater emphasis be placed on determining critical densities or critical proportions, and that more emphasis be placed on developing sampling procedures that will produce reliable results and be adopted by practitioners. These proposals are based on inductively inferring that the distribution of sample observations and some of the parameters that define stop boundaries have only a modest effect on the OC functions for classification sample plans. Because induction is used, exceptions may exist to the generalizations made.
Nonetheless, we feel that our proposal is a good starting point, and the authenticity of the approach can be easily checked using simulation tools (4).

As warrants of proof for the assertions that the distribution of sample observations and some of the parameters that define stop boundaries have only a modest effect on the OC functions for classification sample plans used in pest management, we present analyses of sample plans for three arthropod pests and a disease. Some of the plans are modifications of previously described procedures, whereas others are our own creations based on reported parameters. All of the sample plans are based on Iwao's sequential classification procedure. For each organism, we compare OC and ASN functions when (i) $\alpha$ or maximum sample size is varied, (ii) the sampling distribution is varied, or (iii) the critical density or critical proportion is varied. When the sampling distribution is varied, the purpose is to determine the influence of departures from the distribution used to design the stop boundary on OC and ASN functions. Simulation was used to estimate OC and ASN functions (4).

\section{ANALYSIS OF FOUR SAMPLE PLANS}

The first sample plan analyzed is for alfalfa weevil (Hypera postica (Gyllenhal)). Critical densities for this pest range from 0.5 to 4.5 per plant (1). Legg et al. (10) proposed a sample unit of six stems taken from a $100-\mathrm{m}^{2}$ area and suggested that a minimum of five such samples be taken in each field. The distribution of counts from six stems taken from an approximately $1-\mathrm{m}^{2}$ area can be described by a negative binomial distribution (11). While not explicitly determined, counts from six stems taken from $100 \mathrm{~m}^{2}$ are most likely also distributed according to a negative binomial model. However, the variances among sample observations are less than those when the stems are taken from $1 \mathrm{~m}^{2}$, probably because the six stems are drawn from a larger sample universe, which would tend to reduce the effect of larval aggregation on the sample variance. The variance $\left(s^{2}\right)$ of the six-stem sample counts taken from a $100-\mathrm{m}^{2}$ area can be expressed as a function of the mean as $s^{2}=1.0 \hat{\mu}^{1.2}(1)$.

A nominal sample plan was developed using this variance-mean relationship and the following sample plan parameters: critical density $(c d)=15$ ( 2.5 per plant $\times 6$ plants $), \alpha=0.05$, minimum sample size $(n)=5$, and maximum $n=25$. OC and ASN functions were determined by simulating sample counts as negative binomialdistributed random variables. Three comparisons to the nominal OC and ASN functions were made. In the first, the maximum sample

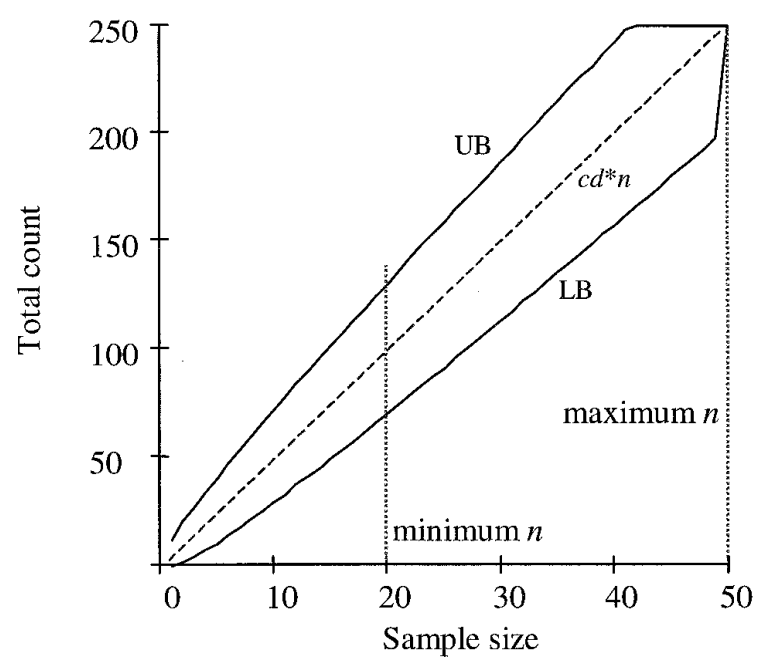

Fig. 3. Stop boundary for Iwao's decision guide. Upper $(U B)$ and lower $(L B)$ boundaries are defined as

$$
U B_{n}=n *\left(c d+z_{\alpha} * \frac{s}{\sqrt{n}}\right) \text { and } L B_{n}=n *\left(c d-z_{\alpha} * \frac{s}{\sqrt{n}}\right) \text {. }
$$


size was varied $(15,25$, and 35$)$; in the second, the parameter $a$ of TPL was varied $(0.5,1.0$, and 1.5$)$; and in the third, $c d$ was varied (11, 15, and 18) (Fig. 4A, B, and C, respectively). Only parameter $a$ of TPL was varied, because linearization of the model $\left(\ln s^{2}=\ln a+b \ln \hat{\mu}\right)$ for fitting via regression leads to error about the regression being incorporated by adding or subtracting quantities to $\ln a$.

Increasing the maximum sample size resulted in larger ASN values but in a negligible change in the OC functions. Thus, as long as the minimum sample size of 5 provided a representative result, there is probably little justification for using a maximum sample size larger than 15. When the TPL parameter $a$ for the simulated data counts was greater than that used to construct the stop boundary, the OC function became flatter and ASN values were reduced. This is because the greater variability among sample counts allowed for quicker crossing of the stop boundaries. The effect of variation in the TPL parameter was minimal for both the OC and ASN functions. Thus, precise knowledge of TPL is probably not important when designing a sample plan for alfalfa weevil. The parameter that had the greatest effect on OC and ASN functions was the critical density, $c d$. A $20 \%$ change in this parameter caused very large changes in $\mathrm{OC}$ values for some true means. For example, with a true density of 15 , the OC for the nominal plan $(c d=15)$ is about 0.5 , meaning that when a population with this mean is
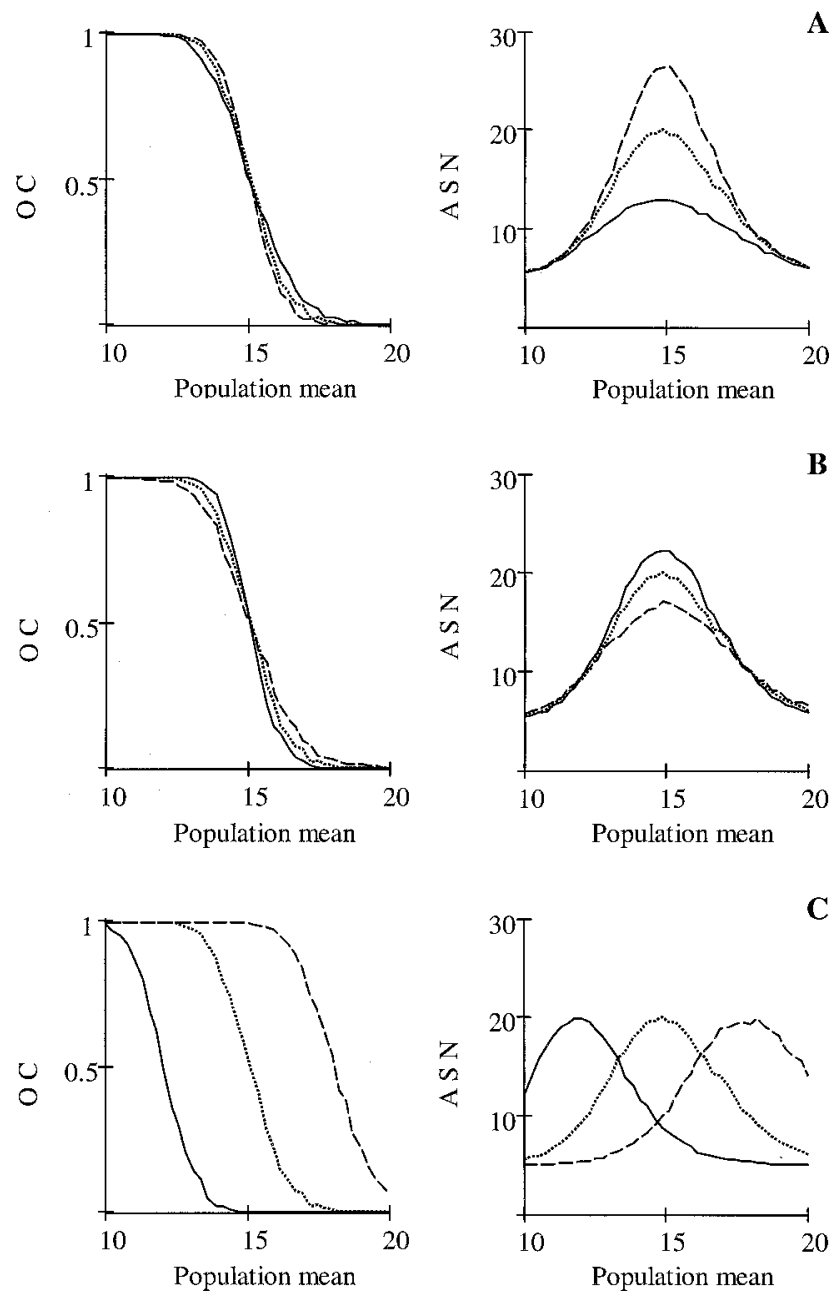

Fig. 4. Influence of sample plan parameters on operating characteristic (OC) and average sample number (ASN) functions for a sample plan based on Iwao's sequential classification decision rule. Nominal parameter values were critical density $(c d)=15$, minimum $n=5$, maximum $n=25$, Taylor's power law (TPL) $a=1.0$, TPL $b=1.2$, and $\alpha=0.05$. A, Maximum $n$ was varied: 15 (solid line), 25 (dotted line), or 35 (dashed line); B, TPL $a$ of simulated counts was varied: 0.5 (solid), 1.0 (dot), or 1.5 (dash); and $\mathbf{C}, c d$ was varied: 12 (solid), 15 (dot), or 18 (dash). sampled, decisions to treat would be made about $50 \%$ of the time. If, however, the true $c d$ was 12 , an incorrect decision to not treat should rarely be made (based on the OC value for the sample plan with a $c d$ of 12), but would be made $50 \%$ of the time when using the sample plan based on a $c d$ of 15 . Similarly, if the true $c d$ was 18 , an incorrect decision to treat should rarely be made when sampling a true density of 15 , but would actually be made around $50 \%$ of the time when using the plan based on a $c d$ of 15 . Thus, of the parameters for this sample plan, $c d$ is most important in terms if its influence on the OC function and, hence, its influence on IPM decision making.

The second plan studied was first described by Vincelli and Lorbeer (17) for use in determining when fungicide applications should be started for control of Botrytis leaf blight of onion caused by Botrytis squamosa. The sampling unit was the three oldest leaves on a plant, and the critical density was three disease lesions per sample unit. When this density is reached, fungicide applications are to be initiated. Counts of lesions per plant can be described using a negative binomial distribution. Vincelli and Lorbeer modeled the variance as a function of the mean using a different model than TPL. We estimated TPL parameters by computing the variance as a function of the mean using the model reported by Vincelli and
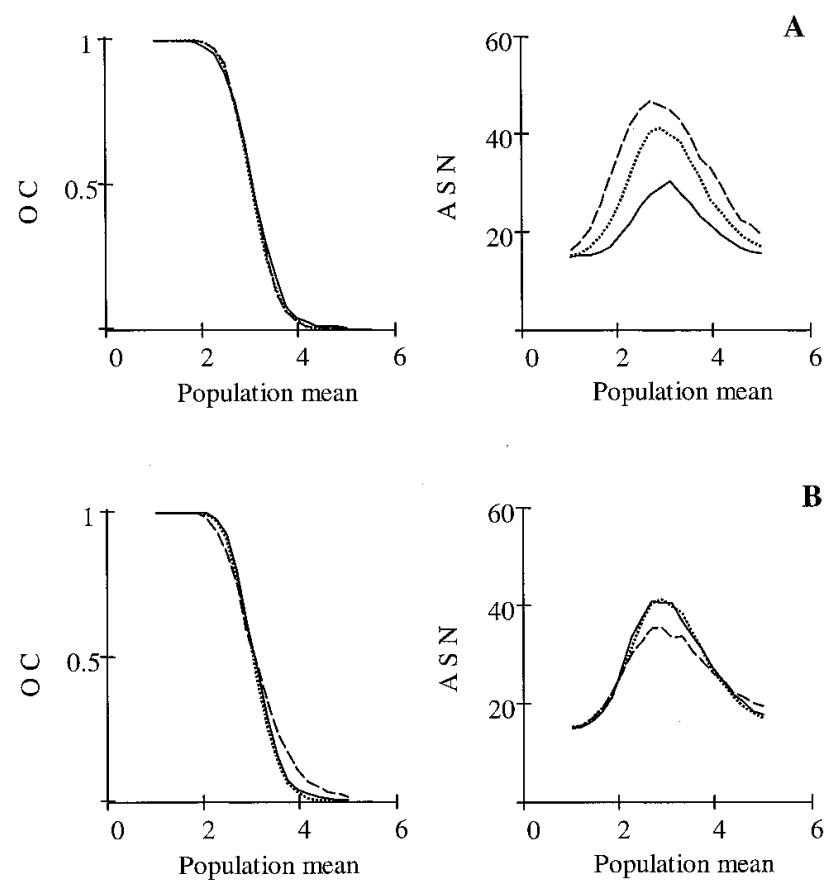

B
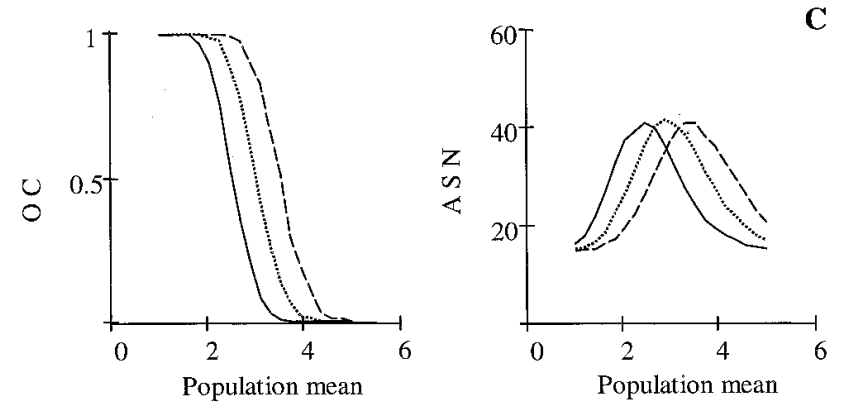

Fig. 5. Influence of sample plan parameters on operating characteristic (OC) and average sample number (ASN) functions for a sample plan based on Iwao's sequential classification decision rule. Nominal parameter values were critical density $(c d)=3$, minimum $n=15$, maximum $n=50$, Taylor's power law (TPL) $a=3.05$, TPL $b=1.02$, and $\alpha=0.05$. A, $\alpha$ was varied: 0.15 (solid line), 0.05 (dotted line), and 0.0125 (dashed line); $\mathbf{B}$, the variance of simulated sample counts was varied: negative binomial $k=1.4$ (solid), TPL used (dot), and $k=0.7$ (dash); and $\mathbf{C}$, $c d$ was varied: 2.5 (solid), 3 (dot), and 3.5 (dash). 
Lorbeer and then fitting TPL to these values using regression. The estimate of $a$ was 3.05 and $b$ was 1.02 . Nominal sample plan parameters were $c d=3$, minimum $n=15$, maximum $n=50$, and $\alpha=$ 0.05 . OC and ASN functions were determined for three values of $\alpha(0.15,0.05$, and 0.0125$)$, three models for the variance of simulated sample counts (negative binomial $k=1.4$, nominal TPL parameters, and $k=0.7)$, and three critical densities (2.5, 3, and 3.5) (Fig. 5). Negative binomial $k=1.4$ was determined by calculating the variance using TPL when the density was equal to $c d$ and then calculating $k$ from this variance. Negative binomial $k=0.7$ simply represented a rather large increase in the variance for densities around $c d$.

As with the alfalfa weevil, changing the sample size (via $\alpha$ instead of maximum $n$ ) or changing the variance of the sample counts applied to the stop boundary had little effect on the OC function (Fig. 5A and B). And, as with the first analysis, departure of the true $c d$ from the $c d$ used to construct the sample plan (the nominal $c d$ ) had a dramatic effect on the OC function (Fig. 5C).

The third sample plan studied was one for classifying lepidopteran pests of fresh market sweet corn in New York. Hoffman et al. (7) showed that when plants were sampled in groups of five, the sampling distribution of plants infested with caterpillars could be described by a beta-binomial distribution. They (7) reported the me-
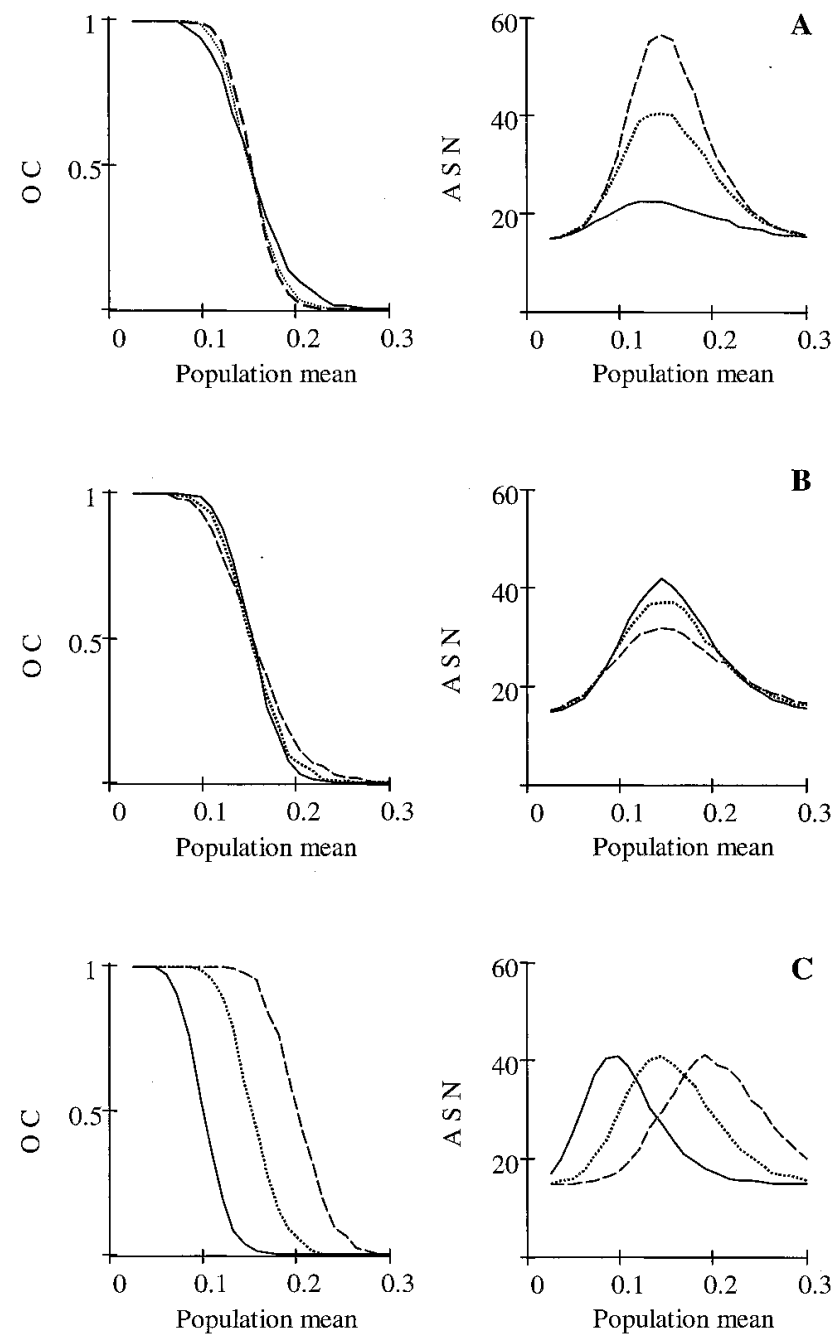

Fig. 6. Influence of sample plan parameters on operating characteristic (OC) and average sample number (ASN) functions for a sample plan based on Iwao's sequential classification decision rule. Nominal parameter values were critical proportion $(c p)=0.15$, minimum $n=15$, maximum $n=50, \rho=0.09$, and $\alpha=0.05$. A, Maximum $n$ was varied: 25 (solid line), 50 (dotted line), and 75 (dashed line); $\mathbf{B}$, the variance of simulated sample counts was varied: $\rho=0.09$ (solid), $\rho=0.22$ ( dot), and $\rho=0.44$ (dash); and $\mathbf{C}, c p$ was varied: 0.1 (solid), 0.15 (dot), and 0.2 (dash). dian value for the aggregation parameter $\rho$ of this distribution to be 0.09 and 90 th and 95 th percentiles to be 0.22 and 0.44 , respectively. As $\rho$ increases, the variance of sample observations increases because sample units (plants) within a sample group (five plants) are more correlated. The critical proportion $(c p)$ of plants infested with larvae was 0.15 prior to silking of the corn and 0.05 after silking. In this analysis, we used $c p=0.15$. The nominal sample plan used minimum $n=15$, maximum $n=50$, and $\alpha=0.05$. OC and ASN functions were determined for three maximum sample sizes $(25,50$, and 75$)$, for three models for the variance of simulated sample counts $(\rho=0.09,0.22$, and 0.44$)$, and for three critical proportions (0.1, 0.15, and 0.2) (Fig. 6).

The patterns obtained are similar to those for the previous two sample plans. Increasing sample size beyond 50 had almost no effect on the OC function, primarily because the variance of the estimated proportion decreases by the square root of the sample size (Fig. 6A). Increasing the variance of sample counts caused the OC functions to be shallower, especially for the more extreme value of $\rho(0.44)$, but the change from the nominal value is not great (Fig. 6B). On the other hand, changing $c d$ resulted in dramatic changes in the OC function (Fig. 6C). If the true $c p$ was 0.1 or 0.2 rather than 0.15 , use of the sample plan based on $c d=0.15$ would result in decision errors $50 \%$ of the time when the true population density was equal to 0.15 . This is in contrast to an error rate of about $10 \%$ if a $c p$ of 0.1 or 0.2 had been used in the sample plan.

The final sample plan analyzed is based on sampling European red mite (Panonychus ulmi (Koch)) in apples. Nyrop and Binns (13) described critical densities for use in monitoring mite densities and provided descriptions of the sampling distribution of motile mites on intermediate-aged apple leaves. These sample counts could be described using a negative binomial distribution with the variance modeled as a function of the mean using TPL ( $a=4.3, b=$ 1.4). Jones (9) reviewed TPL parameters estimated for two Panonychus species and suggested that generic parameters of $a=3.3$ and $b=1.32$ be used when constructing sample plans. We used these values to construct the nominal sample plan as well as $c d=5$,
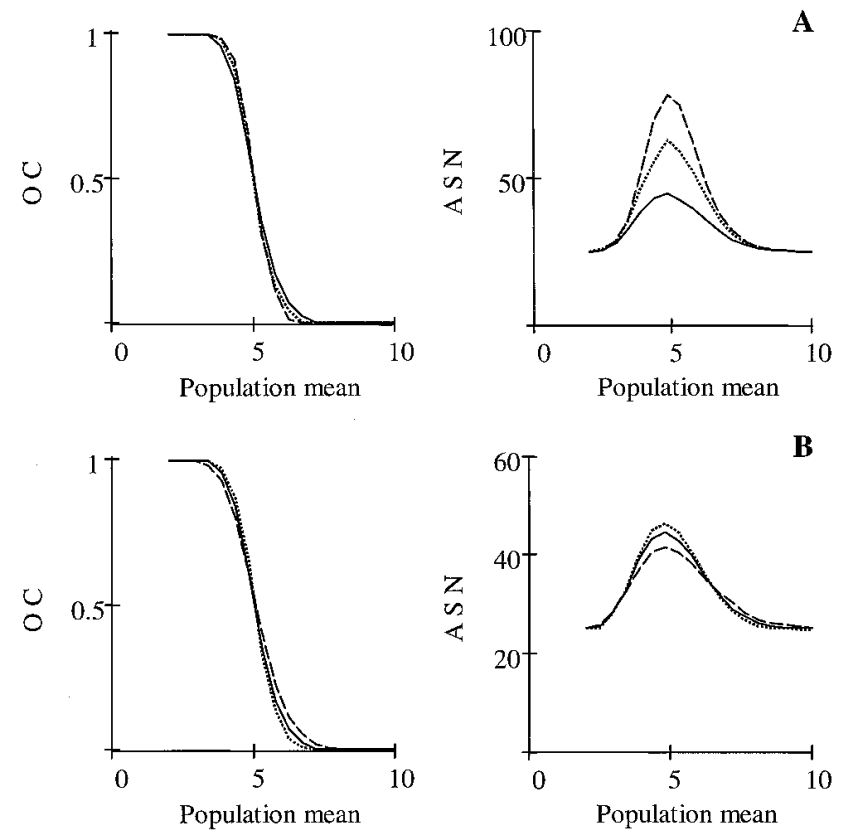

Fig. 7. Influence of sample plan parameters on operating characteristic (OC) and average sample number (ASN) functions for a sample plan based on Iwao's sequential classification decision rule. Nominal parameter values were critical density $(c d)=2.5$, Taylor's power law (TPL) $a=3.3, b=1.32$, minimum $n=25$, maximum $n=50$, and $\alpha=0.05$. A, Maximum $n$ was varied: 50 (solid line), 75 (dotted line), and 100 (dashed line). B, TPL parameters for simulated sample counts were varied: $a=3.3, b=1.32$ (solid); $a=4.3, b=$ 1.4 (dot); and $a=2.8, b=1.22$ (dash). 
minimum $n=25$, maximum $n=50$, and $\alpha=0.05$. OC and ASN functions were determined for three maximum sample sizes (50, 75, and 100) and for three TPL models for the variance of simulated sample counts ( $a=3.3, b=1.32$ (nominal); $a=2.8, b=$ 1.22 ; and $a=4.3, b=1.4$ ) (Fig. 7). The second set of TPL parameters was one standard deviation less than the nominal values (9), and the third set of TPL parameters was that estimated for European red mite by Nyrop and Binns (13). The critical density was not altered because, by now, it is clear that this parameter will have a large effect on the OC and ASN functions.

Increasing the maximum sample size, even doubling it, had a very modest influence on the $\mathrm{OC}$ function (Fig. 7A). Increasing the sample variance caused the OC function to be shallower, and decreasing the sample variance caused the OC function to be steeper (Fig. 7B). The changes were very slight, however, and use of the generic parameters would produce quite acceptable results.

Sampling small and often abundant organisms like spider mites is difficult, and to overcome this snarl, binomial counts are often used in lieu of complete enumeration. With binomial counts, samples are scored as positive if the number of organisms in a sample unit exceed a tally threshold, $T$, and zero otherwise. The estimated proportion of positive scores $\left(p_{T}\right)$ is then used to estimate density. To do so, a model is used to translate the proportion of positive binomial counts into density. One way this can be done is by using a probability distribution function, such as the negative binomial distribution (9). For European red mite, the $p_{T}-\hat{\mu}$ model consists of the negative binomial distribution with TPL used to model the relationship between the parameter $k$ and the mean density. The $p_{T}-\hat{\mu}$ model is rather sensitive to changes in $k$, so OC functions for binomial count sample plans may be more sensitive to changes in TPL parameters than are complete enumeration sample plans.

To test this hypothesis, we constructed fixed sample size plans $(n=50)$ to classify density about a critical density of 5 . We constructed plans using two tally thresholds, 0 and 5. Using a tally threshold close to the critical density makes the sample plan more robust to departures from the nominal $p_{T}-\hat{\mu}$ model (3). Variation about the nominal model comes from two sources: from differ-

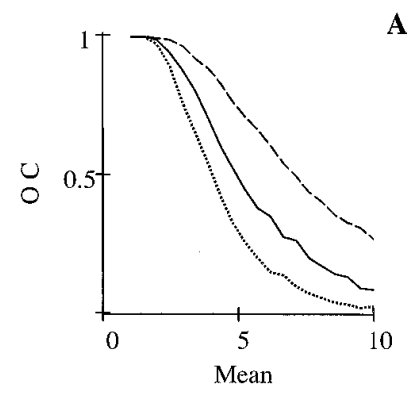

$\mathbf{A}$
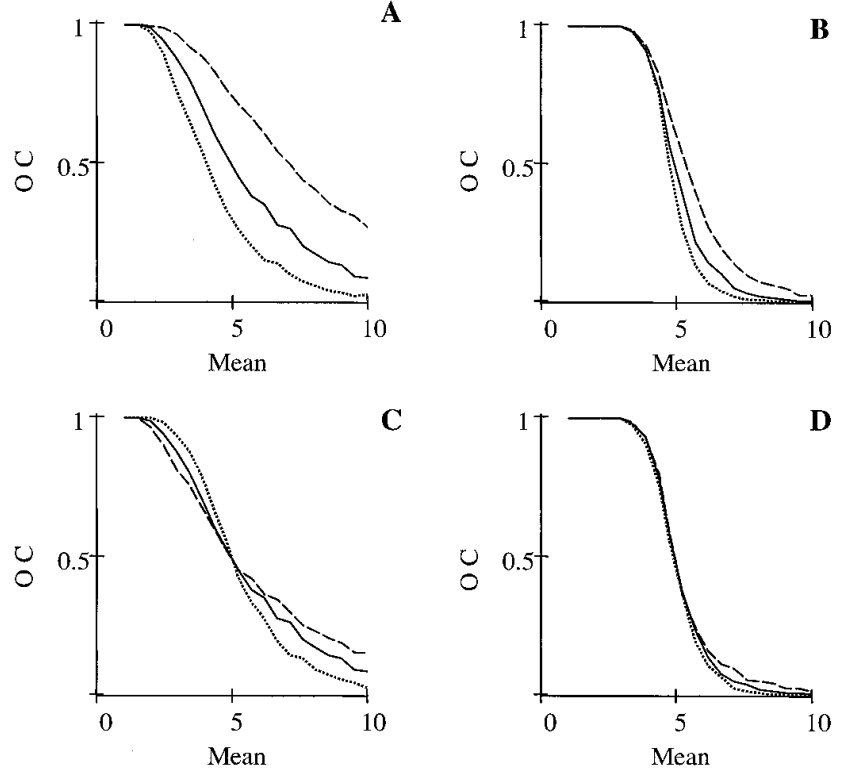

C

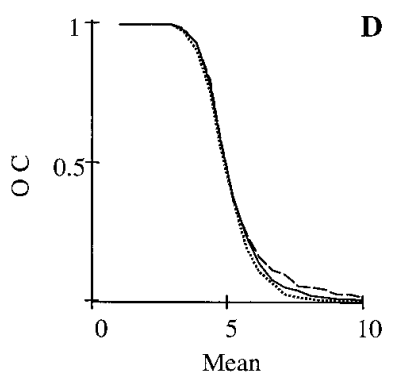

Fig. 8. Operating characteristic (OC) functions for fixed sample size $(n=50)$ binomial count sampling plans used to classify density with respect to a critical density of 5 . A and B, Three sets of Taylor's power law (TPL) parameters were used ( $a=3.3, b=1.32$ [solid line]); $a=2.8, b=1.22$ [dotted line]; and $a=4.3, b=1.4$ [dashed line]) with a tally number $(T)$ of either $\mathbf{A}, 0$ or $\mathbf{B}, 5$ and mean square error (mse) from TPL regression $=0.55$. $\mathbf{C}$ and $\mathbf{D}$, One set of TPL parameters was used $(a=3.3, b=1.32)$ with three values for mse $(0.55$ [solid line], 0.35 [dotted line], and 0.75 [dashed line]) and a tally number $(T)$ of either C, 0 or $\mathbf{D}, 5$. ences in model parameters (e.g., $a=3.3, b=1.32$ versus $a=4.3$, $b=1.4$ ) and from variation about the TPL line defined by the parameters. The variation about the line described by the TPL parameters can be modeled using the variance (mean square error [ $\mathrm{mse}]$ ) from the regression used to estimate the TPL parameters (13). This variation always occurs and results in the OC functions for binomial count sample plans being shallower than if only binomial variation was used to estimate the OC functions (13).

OC functions were determined using simulation (4) for four combinations of TPL parameters and tally number (Fig. 8A and B). In the first two simulations, OC functions were compared for the three sets of TPL parameters ( $a=3.3, b=1.32 ; a=2.8, b=1.22$; and $a=4.3, b=1.4)$ when the tally number $(T)$ was either 0 or 5 . The mse for TPL reported by Nyrop and Binns (13), 0.55, was used. When $T=0$, departure in the TPL parameters from the nominal values produced large changes in the $\mathrm{OC}$ functions. The parameters reported by Nyrop and Binns (13) caused the OC function to be both shallower and shifted to right on the mean density axis compared with the nominal OC functions. This occurs because the larger TPL parameters cause the $p_{T}-\hat{\mu}$ model to also be shallower and shifted to the right on the mean density axis. In contrast, the smaller TPL parameters caused the OC function to be both steeper and shifted to the left because the $p_{T}-\hat{\mu}$ model is steeper and shifted to the left on the mean density axis. Using a tally number of 5 greatly ameliorated these effects, but did not eliminate them. The results obtained with a tally number of 5 occurred because this tally number causes the $p_{T}-\hat{\mu}$ model for any of the three sets of TPL parameters to have approximately the same midpoints (e.g., $p_{T}$ approximately 0.5 ) when the true density is 5 .

The estimated variance about the TPL parameters also influences the OC functions for binomial count models. Increasing the variance causes the OC function to be shallower, and decreasing the variance causes the OC function to be steeper. Jones (9) did not report estimated mse values, so in the second simulation, we compared OC functions for mse values distributed about the estimate reported by Nyrop and Binns (1992): 0.35, 0.55, and 0.75 (Fig. 8C and D). Increasing the tally number from 0 to 5 greatly reduced the differences among the OC functions for reasons analogous to those provided when the TPL parameters themselves changed. The variance about the TPL model is used to describe a family of $p_{T}-\hat{\mu}$ models about the nominal $p_{T}-\hat{\mu}$ model defined by the TPL function. Increasing the tally number to 5 causes this family of $p_{T}-\hat{\mu}$ models to all have midpoints at about a density of 5 .

To summarize this section on binomial count models, departures from the nominal TPL parameters can cause rather large changes in the OC functions. This is in contrast to when complete enumeration is used. However, by using a tally number close to $c d$, these effects can be greatly reduced. For the European red mite example, use of the generic TPL parameters with a tally number of 5 would provide acceptable results.

\section{CONCLUSIONS AND RECOMMENDATIONS}

Based on the sample plans investigated, three conclusions can be drawn regarding the influence of sample plan parameters on $\mathrm{OC}$ and ASN functions. First, the critical density or critical proportion has the greatest influence on the OC function and, hence, also on the proportion of correct and incorrect management decisions. Quite often, these parameters are the least well known when developing sample plans for use in IPM decision making. The second conclusion is that changes in the sampling distribution have only a very modest influence on OC functions for complete count sample plans. This result is somewhat dependent on the fact that a minimum number of samples was always examined before making a decision on the need for further samples. However, this is not a shortcoming, because a minimum number of samples will usually be taken when sampling pests for the purpose of control decision making. For binomial count plans, parameters for the $p_{T}-\hat{\mu}$ model 
could greatly influence the OC function. However, this effect was ameliorated by having the tally threshold about equal to the critical density. The third conclusion is that the OC function is relatively insensitive to continued increases in sample size. It is not possible to know a priori at what point further increases in sample size produce increasingly modest improvements in OC, but this is easily determined using simulation.

Based on these conclusions, we make three recommendations for developing sample plans for use in pest management decision making. Ideally, the first step in developing a sample plan should be to obtain a good estimate of $c d$ or $c p$. Of course, this may not be possible, and it is often the case that using some value for these parameters, even though not the theoretically correct value, would markedly improve IPM decision making. It is then important to recognize the vagueness of $c d$ or $c p$ and understand that precise information about pest abundance provides a false sense of security. In such cases, reductions in decision errors will come from improved knowledge of $c p$ or $c d$ and not from increasing the precision of the sample information.

The second recommendation is that, when developing sampling plans, one should start by determining what is known about the sampling distribution, use this information to develop a sample plan, and then perform a sensitivity analysis to determine if further refinement of this information is warranted. When such information is not available, sampling distribution models might be grouped for similar taxa on similar crops (e.g., aphids on small grains, disease incidence on perennial fruit crops). This hypothesis is easily tested using simulation.

The third recommendation is that sample size should first be based on the sample information being representative. This means that the sample information is a good indicator of pest abundance or incidence in the management unit. Sample information may not be representative if sample observations are not collected from throughout the sample universe, which can happen when very few samples are taken.

Information on pest abundance is a key ingredient of many pest management programs. Considerable effort is often expended to develop strategies for collecting this information. For this purpose, precise descriptions of sampling distributions are often unnecessary and generic descriptions may suffice. Specification of sample size so that some arbitrary precision in the sample information is obtained is usually unnecessary. Rather, minimal sample sizes should be established so that the risks of incorrect decisions are acceptable and sample plans designed so that the sample information is representative of the pest population. Greater emphasis should be placed on characterizing pest densities that signal the need for management intervention.

\section{LITERATURE CITED}

1. Barney, R. J., and Legg, D. E. 1987. Accuracy of a single 30-stem sample for detecting alfalfa weevil larvae (Coleoptera: Curculionidae) and making management decisions. J. Econ. Entomol. 80:512-516.

2. Binns, M. R. 1994. Sequential sampling for classifying pest status. Pages 137-174 in: Handbook of Sampling Methods for Arthropods in Agriculture. L. P. Pedigo and G. D. Buntin, eds. CRC Press, Boca Raton, FL.

3. Binns, M. R., and Bostanian, N. J. 1990. Robust binomial decision rules for integrated pest management based on the negative binomial distribution. Am. Entomol. 36:50-54.

4. Binns, M. R., Nyrop, J. P., and van der Werf, W. Sampling and Monitoring in Crop Protection: The Theoretical Basis for Designing Practical Decision Guides. CAB International, Wallingford, United Kingdom. In press.

5. Elliott, N. C., Hein, G. L., and Shepard, B. M. 1994. Sampling arthropod pests of wheat and rice. Pages 627-666 in: Handbook of Sampling Methods for Arthropods in Agriculture. L. P. Pedigo and G. D. Buntin, eds. CRC Press, Boca Raton, FL.

6. Green, R. H. 1970. On fixed precision level sequential sampling. Res. Popul. Ecol. 12:249-251.

7. Hoffman, M. P., Nyrop, J. P., Kirkwyland, J. J., Riggs, D. M., Gilrein, D. O., and Moyer, D. D. 1996. Sequential sampling plan for scheduling control of lepidopteran pests of fresh market sweet corn. J. Econ. Entomol. 89:386-395.

8. Iwao, S. 1975. A new method of sequential sampling to classify populations relative to a critical density. Res. Popul. Ecol. 16:281-288.

9. Jones, V. P. 1990. Developing sampling plans for spider mites (Acari: Tetranychidae): Those who don't remember the past may have to repeat it. J. Econ. Entomol. 83:1656-1664.

10. Legg, D. E., Shufran, K. A., and Yeargan, K. V. 1985. Evaluation of two sampling methods for their influence on the population statistics of alfalfa weevil (Coleoptera: Curculionidae) larva infestations in alfalfa. J. Econ. Entomol. 78:1468-1474.

11. Miller, C. D. F., Mukerji, M. K., and Guppy, J. C. 1972. Notes on the spatial pattern of Hypera postica (Coleoptera: Curculionidae) on alfalfa. Can. Entomol. 104:1995-1999.

12. Naranjo, S. E., and Hutchinson, W. D. 1997. Validation of arthropod sampling plans using a resampling approach: Software and analysis. Am. Entomol. 43:48-57.

13. Nyrop, J. P., and Binns, M. R. 1992. Algorithms for computing operating characteristic and average sample number functions for sequential sampling plans based on binomial count models and revised plans for European red mite (Acari: Tetranychidae) on apple. J. Econ. Entomol. 85: 1253-1273.

14. Pedigo, L. P., and Buntin, G. D., eds. 1994. Handbook of Sampling Methods for Arthropods in Agriculture. CRC Press, Boca Raton, FL.

15. Southwood, T. R. E. 1978. Ecological Methods with Particular Reference to the Study of Insect Populations. Chapman and Hall, London.

16. Taylor, L. R. 1961. Aggregation, variance, and the mean. Nature 189:732.

17. Vincelli, P. C., and Lorbeer, J. W. 1987. Sequential sampling plan for timing initial fungicide application to control Botrytis leaf blight of onion. Phytopathology 77:1301-1303. 